\title{
Application of Long Short-Term Memory for Faults Identification in 3-Phase Induction Motor
}

\author{
Md Motaher Hossain ${ }^{1}$, Sri R. Kolla ${ }^{2}$ \\ Student, Electronics and Computer Engineering Technology, Bowling Green State University, Bowling Green, USA ${ }^{1}$ \\ Professor, Electronics and Computer Engineering Technology, Bowling Green State University, Bowling Green, USA ${ }^{2}$
}

\begin{abstract}
This paper presents a Long Short-Term Memory (LSTM) network-based method to identify faults in a 3-phase induction motor. Various external faults in a 3-phase induction motor are first described. A brief introduction to LSTM technique is then presented. The LSTM is trained to classify external faults using 3-phase voltages and currents collected from a $1 / 3 \mathrm{hp}$ induction motor in real-time. MATLAB is used for training and testing the LSTM method. Results show that the proposed LSTM based method is effective in classifying different external faults in the 3-phase induction motor. The performance of the LSTM method is observed to be better than some of the previous methods in model formation and testing accuracy.
\end{abstract}

Keywords: Long Short-Term Memory, Induction Motor, Fault Identification, Machine Learning, Protective Relays.

\section{INTRODUCTION}

The 3-phase induction motor is the most important motor used in industries. The operating conditions may sometimes lead the motor into different fault conditions. The major external faults range from overload to single phasing [1]. To avoid damage, the motor should be disconnected when a fault is experienced. Microcomputer-based protective relays monitor the motor and disconnect it when a fault occurs [2]. The relay logic used to identify these faults requires effective signal processing techniques for reliable and fast operation. With the recent developments in Machine Learning (ML), methods such as Artificial Neural Networks (ANN), Support Vector Machines (SVM) and Deep Learning Techniques (DLT) have been currently applied in induction motor's faults detection [3, 4]. The conventional neural networks approach may produce models that can over-fit the data due to limitations with generalization. The SVM method showed better models for identifying faults. Recently DLT have been extensively used in different engineering applications including faults identification [4,5]. The Long Short-Term Memory (LSTM) network is one of the DLT received considerable attention.

Siddique et al. [6]and Ojaghi et al. [7] presented various stator fault identification methods for induction motors in their review papers. Many researchers have used the Motor Current Signature Analysis (MCSA) method that uses high frequency components in current to diagnose faults in induction motors [8]. Incipient faults such as stator winding faults and bearing wear in single-phase induction motors were detected using current and speed as inputs to a neural network by Chow and Yee [9]. In reference [10], ANN was used for external faults classification in real-time for a 3-phase induction motor. The SVM method for detecting faults in induction motors was proposed by Poyhonen et al. [11], Salem et al. [12] and Kolla and Hammo [3]. A motor protection system based on fuzzy logic was proposed in [13]. The SVM for identifying broken rotor bars in motors was used by Keskes et al. [14]. The SVM and MCSA methods were combined to detect induction motor faults by Fang and Ma [15]. Recently deep learning methods such as Convolution Neural Network (CNN) and LSTM were applied to mechanical faults diagnosis in rotating machines including induction motor [16-20]. Pan et al. used CNN and LSTM to diagnose bearing faults in a motor dynamometer system [16]. Eren has also applied CNN for detecting faults in bearings [17]. Lee et al. applied CNN to diagnose rotor and bearing faults in induction motors [18]. Yang et al. used LSTM for detecting mechanical faults in the wind turbine drivetrain diagnostics simulator [19]. Lei et al. also used LSTM for mechanical faults diagnosis in an experimental wind turbine system [20]. However, there was not much work done in applying deep learning techniques to detect external electrical faults in 3-phase induction motors.

This paper presents LSTM-based technique to detect external electrical faults in a 3-phase induction motor. Different types of external faults in an induction motor are explained in Section II. A review of LSTM network is presented in Section III. The LSTM is trained to identify faults using 3-phase RMS currents and voltages obtained in real-time from a $1 / 3 \mathrm{hp}$ cage rotor induction motor. MATLAB program is used for training the LSTM network. These details are described in Section IV. The trained LSTM is tested with fault currents and voltages data from the induction motor. These testing results are discussed in Section V. Concluding remarks are offered in Section VI. 


\section{FAULTS IN 3-PHASE INDUCTION MOTOR}

Different types of external electrical fault conditions are generally experienced by a 3-phase induction motor [1]. The faults include overload, locked rotor, over voltage, under voltage, unbalanced supply voltage, and single phasing. Protective relays monitor the motor to disconnect when these fault occur [2]. These faults and protection used for them were described in references $[1,3]$. The motor voltages and currents have distinctive features during faults as discussed in $[3,10]$. As an example, 3-phase currents and voltages for an unbalanced voltage fault are shown in Fig. 1. It can be noted that the three voltages and currents have unequal amplitudes and phase angles. These characteristics are used by relaying methods to identify various faults in induction motors [1].

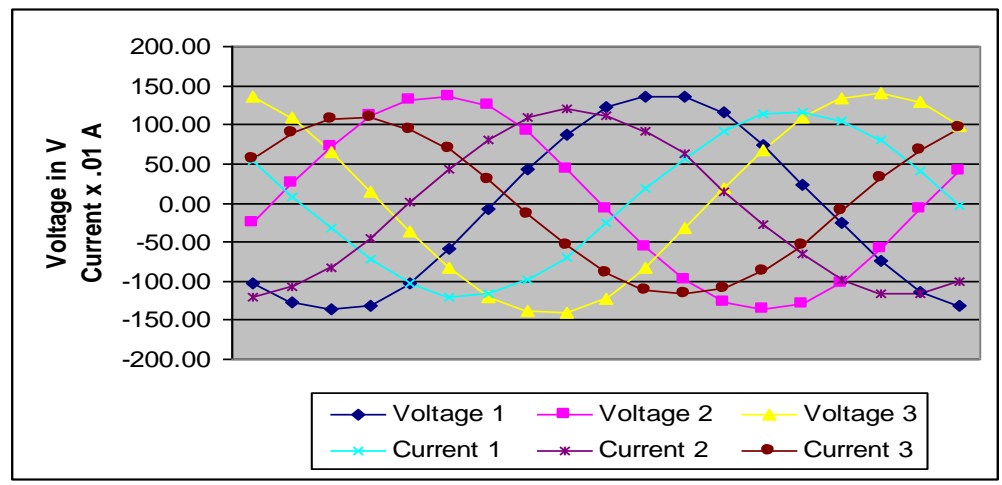

Fig. 13 -phase voltages and currents for unbalanced voltage fault

Microcomputer-based relay methods were proposed in the literature to protect a motor during external fault conditions $[1,2]$. There have been recent attempts to use ML techniques such as ANN and SVM to classify these faults [3, 10]. This paper further investigates the use of ML techniques, and applies LSTM-based method to detect external faults in 3phase induction motors.

\section{LONG SHORT-TERM MEMORY}

In the past, the LSTM network was successfully used in the deep learning techniques to overcome the problems associated with vanishing and exploding gradients in basic Recurrent Neural Network (RNN) [21, 22]. The LSTM was proposed in 1997 by Hochreiter and Schmidhuber [21] and improved in 1999 by Gers et al. [22]. The intuition behind LSTM is to remember all the previous knowledge that the network is engaged so far and forget all the irrelevant data. These are accomplished by introducing a LSTM block in the RNN. Specifically, different activation function layers are used for different purposes to achieve the objectives of LSTM [23].

A basic LSTM structure has four different gates called input gate, output gate, forget gate, and a cell unit which are shown in Fig. 2 [23]. The working principle of LSTM is similar to the operation of the RNN [21]. However, the internal cell state of LSTM is passed forward along with the hidden state that differs from the RNN [23].

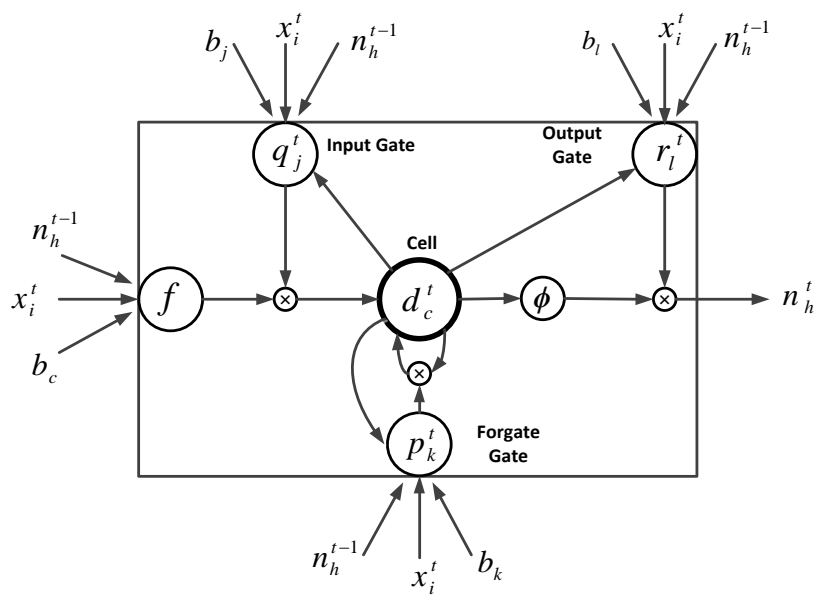

Fig. 2 The basic architecture of a peephole LSTM cell 


\section{DOI 10.17148/IJIREEICE.2020.8901}

The algorithms of LSTM can be expressed from Fig. 2 and given by the following equations [23],

$$
\begin{aligned}
& \mathrm{q}_{\mathrm{j}}^{\mathrm{t}}=\sum_{\mathrm{i}=1}^{\mathrm{I}} \mathrm{w}_{\mathrm{ij}} \mathrm{x}_{\mathrm{i}}^{\mathrm{t}}+\sum_{\mathrm{h}=1}^{\mathrm{H}} \mathrm{w}_{\mathrm{hj}} \mathrm{n}_{\mathrm{h}}^{\mathrm{t}-1}+\sum_{\mathrm{c}=1}^{\mathrm{C}} \mathrm{w}_{\mathrm{cj}} \mathrm{m}_{\mathrm{c}}^{\mathrm{t}-1}+\mathrm{b}_{\mathrm{j}} \\
& \mathrm{n}_{\mathrm{j}}^{\mathrm{t}}=\mathrm{f}\left(\mathrm{q}_{\mathrm{j}}^{\mathrm{t}}\right) \\
& \mathrm{p}_{\mathrm{k}}^{\mathrm{t}}=\sum_{\mathrm{i}=1}^{\mathrm{I}} \mathrm{w}_{\mathrm{ik}} \mathrm{x}_{\mathrm{i}}^{\mathrm{t}}+\sum_{\mathrm{h}=1}^{\mathrm{H}} \mathrm{w}_{\mathrm{hk}} \mathrm{n}_{\mathrm{h}}^{\mathrm{t}-1}+\sum_{\mathrm{c}=1}^{\mathrm{C}} \mathrm{w}_{\mathrm{ck}} \mathrm{m}_{\mathrm{c}}^{\mathrm{t}-1}+\mathrm{b}_{\mathrm{k}} \\
& \mathrm{n}_{\mathrm{k}}^{\mathrm{t}}=\mathrm{f}\left(\mathrm{p}_{\mathrm{k}}^{\mathrm{t}}\right) \\
& \mathrm{n}_{\mathrm{c}}^{\mathrm{t}}=\sum_{\mathrm{i}=1}^{\mathrm{I}} \mathrm{w}_{\mathrm{ic}} \mathrm{x}_{\mathrm{i}}^{\mathrm{t}}+\sum_{\mathrm{h}=1}^{\mathrm{H}} \mathrm{w}_{\mathrm{hc}} \mathrm{n}_{\mathrm{h}}^{\mathrm{t}-1}+\mathrm{b}_{\mathrm{c}} \\
& \mathrm{d}_{\mathrm{c}}^{\mathrm{t}}=\mathrm{n}_{\mathrm{j}}^{\mathrm{t}} \mathrm{m}_{\mathrm{c}}^{\mathrm{t}-1}+\mathrm{n}_{\mathrm{k}}^{\mathrm{t}} \mathrm{g}\left(\mathrm{n}_{\mathrm{c}}^{\mathrm{t}}\right) \\
& \mathrm{r}_{\mathrm{l}}^{\mathrm{t}}=\sum_{\mathrm{i}=1}^{\mathrm{l}} \mathrm{w}_{\mathrm{il}} \mathrm{x}_{\mathrm{i}}^{\mathrm{t}}+\sum_{\mathrm{h}=1}^{\mathrm{H}} \mathrm{w}_{\mathrm{hl}} \mathrm{n}_{\mathrm{h}}^{\mathrm{t}-1}+\sum_{\mathrm{c}=1}^{\mathrm{C}} \mathrm{w}_{\mathrm{cl}} \mathrm{m}_{\mathrm{c}}^{\mathrm{t}-1}+\mathrm{b}_{\mathrm{l}} \\
& \mathrm{n}_{\mathrm{l}}^{\mathrm{t}}=\mathrm{f}\left(\mathrm{r}_{\mathrm{l}}^{\mathrm{t}}\right) \\
& \mathrm{n}_{\mathrm{h}}^{\mathrm{t}}=\mathrm{n}_{\mathrm{l}}^{\mathrm{t}} \Phi\left(\mathrm{d}_{\mathrm{c}}^{\mathrm{t}}\right)
\end{aligned}
$$

where, the input from cell to input gate is represented by $\mathrm{m}$, the input gate vector number is $\mathrm{j}$, the forget gate vector number is $\mathrm{k}$, the output gate vector number is 1 , the cell vector number is $\mathrm{c}$, the value of the cell is expressed by $\mathrm{d}$, and the activation functions are given by $\mathrm{f}, \mathrm{g}$, and $\Phi$. A typical architecture of LSTM consists of sequence input layer, LSTM layer, fully connected layer, softmax layer, and classification layer. These layers are shown in Fig. 3. A description of some of these layers is given below.

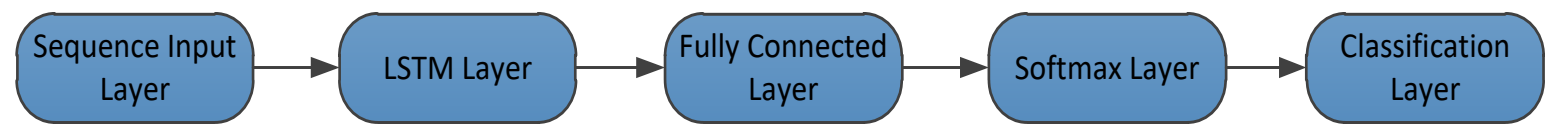

Fig. 3 Proposed LSTM model for fault detection

The fully connected layer takes the output from the previous layer and sends it to the next layer after flattening it and changes into a single vector where every output neuron from the previous layer is connected to every one of the next layer neurons [4]. The Softmax classifier is used for multi-category classifier problems in which an input vector is sent into a mathematical function that produces an output vector whose elements are in the range (0-1), and they add up to 1[4]. The mathematical function is expressed by:

$\mathrm{S}\left(\mathrm{y}_{\mathrm{i}}\right)=\frac{\mathrm{e}^{\mathrm{i} \mathrm{y}}}{\sum_{\mathrm{j}} \mathrm{e}^{\mathrm{jy}}}$

where $S$ is the softmax function for the variable $y$. The derivative of the softmax function is calculated during the back propagation process to update their respective weights in the network [4]. The total error is minimized using these updated weights in gradient calculations. The derivative of softmax function is expressed by:

$\frac{\mathrm{dS}\left(\mathrm{y}_{\mathrm{i}}\right)}{\mathrm{dy}_{\mathrm{i}}}=\mathrm{S}\left(\mathrm{y}_{\mathrm{i}}\right)\left(1-\mathrm{S}\left(\mathrm{y}_{\mathrm{i}}\right)\right)$

In the softmax classifier the cross-entropy loss function is used because it gives a better performance where the output is a probability distribution [4]. The mathematical expression of the cross-entropy loss function L used in regression and classification problems is given by:

$\mathrm{L}(\mathrm{y})=-\sum_{\mathrm{i}} \mathrm{y}_{\mathrm{i}}^{\prime} \log \left(\mathrm{y}_{\mathrm{i}}\right)$

where $y$ and $\mathrm{y}^{\prime}$ are the output label and target label of the network.

In deep learning, an optimizer is required to reduce the loss functions. During the learning process, the optimizer is used to find out the optimized value of weights based on the value of loss function [24]. Adam optimization, used in this paper, is also known as Adaptive Momentum Estimation and is a first-order gradient-based optimization for stochastic objective functions, based on exponential weighted moving averages [24]. It estimates both the first momentum and the second moment of the gradient [24]. Assume that $f(\theta)$ is the stochastic objective function with parameters $\theta$. The first momentum vector and second moment vector can be written as:

$\mu_{\mathrm{t}}=\beta_{1} \cdot \mu_{\mathrm{t}-1}+\left(1-\beta_{1}\right) \cdot \mathrm{g}_{\mathrm{t}}$

$\mathrm{v}_{\mathrm{t}}=\beta_{2} \cdot \mathrm{v}_{\mathrm{t}-1}+\left(1-\beta_{2}\right) \cdot \mathrm{g}_{\mathrm{t}}^{2}$

where, $\beta_{1}$ and $\beta_{2}$ are the weighting parameters whose values are usually 0.9 and 0.999 respectively, $g_{t}$ is the gradient of objective function given by:

$\mathrm{g}_{\mathrm{t}}=\nabla_{\theta} \cdot \mathrm{f}_{\mathrm{t}}\left(\theta_{\mathrm{t}-1}\right)$

The Adam optimizer uses a re-normalization. The corresponding bias-corrected first momentum vector $\hat{\mu}_{t}$ and biascorrected second moment vector $\hat{v}_{\mathrm{t}}$ can be represented as:

$\hat{\mu}_{\mathrm{t}}=\frac{\mu_{\mathrm{t}}}{1-\beta_{1}}$
$\hat{\mathrm{v}}_{\mathrm{t}}=\frac{\mathrm{v}_{\mathrm{t}}}{1-\beta_{2}}$ 
International Journal of Innovative Research in

Electrical, Electronics, Instrumentation and Control Engineering

Vol. 8, Issue 9, September 2020

\section{DOI 10.17148/IJIREEICE.2020.8901}

The updated form of the objective function parameter is given by:

$\theta_{\mathrm{t}}=\theta_{\mathrm{t}-1}-\alpha \cdot \frac{\widehat{\mu}_{\mathrm{t}}}{\sqrt{\widehat{\mathrm{v}}_{\mathrm{t}}}+\epsilon}$

where the learning rate is $\alpha$ and $\epsilon$ is a very small number. The common choices for initialization of the Adam optimization algorithm are $\alpha=0.001, \epsilon=10^{-8}, \mu_{0}=0, v_{0}=0$ and $\mathrm{t}=0$.

\section{ISTM METHOD FOR IDENTIFYING INDUCTION MOTOR FAULTS}

The first step in using the LSTM method for identifying induction motor no fault and fault conditions is to select inputs and outputs for the LSTM method. Inputs are selected as RMS values of 3-phase currents and voltages in this paper. This results in six (6) inputs to LSTM. The data is classified into seven (7) output categories corresponding to no fault and six faults conditions described before. The outputs are categorised from F1 to F7 and the corresponding category is obtained from LSTM if that condition exits. The inputs (RMS currents and voltages) and outputs (no fault and faults conditions) of the LSTM model are shown in Fig. 4.

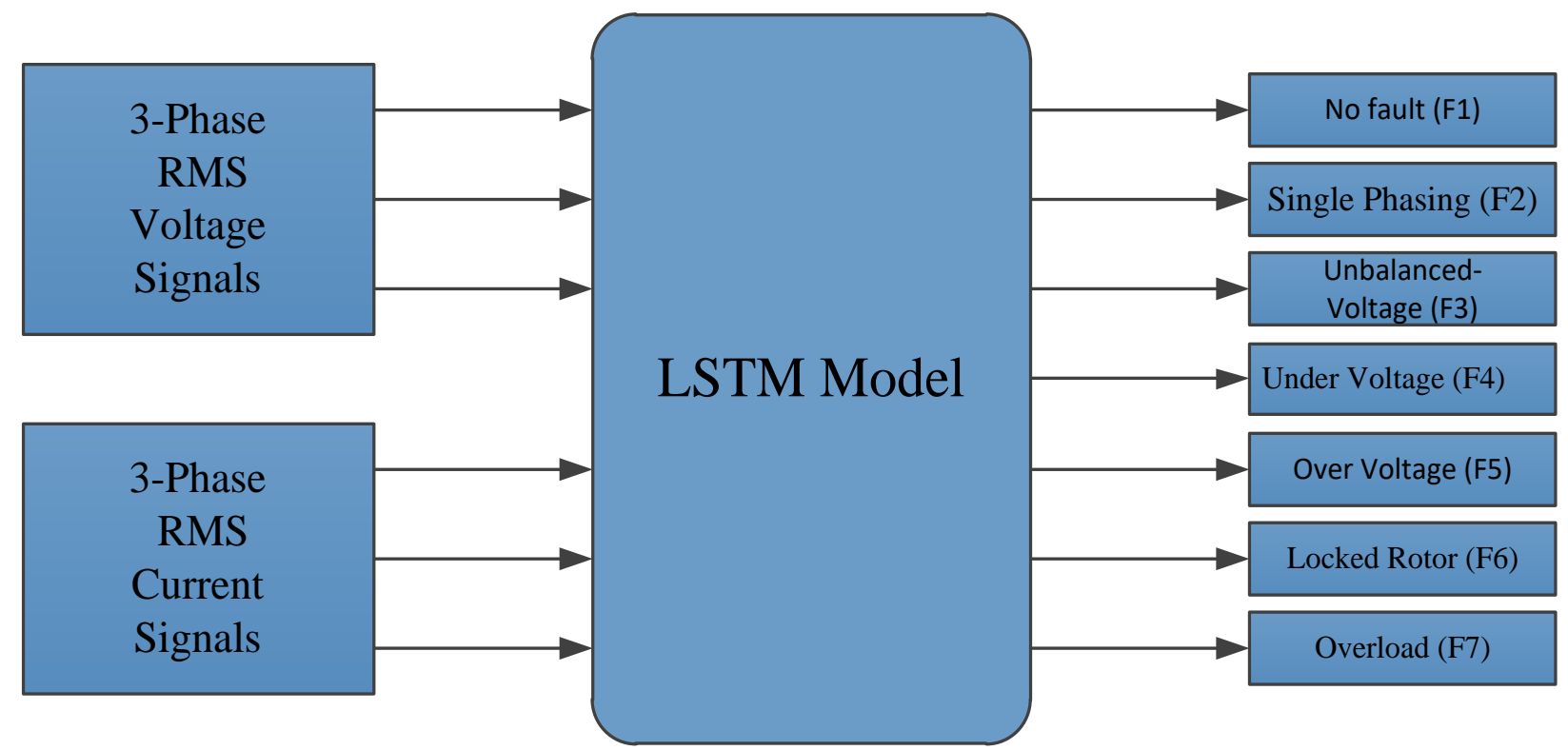

Fig. 4 Inputs and outputs of LSTM method to detect induction motor external faults

Training the LSTM is the next step for detecting fault conditions. The current and voltage waveforms for no fault and six faults condition are used for this training. A $1 / 3 \mathrm{hp}, 208 \mathrm{~V}, 3$-phase cage-rotor induction motor was used to collect these waveforms in real-time [10]. Over voltage, under voltage and unbalanced supply voltage fault conditions were created using three variacs. Single phasing fault was simulated by removing one phase power line connection. A prony brake was used to produce locked rotor and overload fault conditions. These faults data were obtained in real-time by a LabVIEW program [10]. The current and voltage waveforms for a single phasing fault are shown in Fig. 5. It can be noted that one phase current is zero and the currents in other two phases are $180^{\circ}$ apart. For other faults also similar waveforms were obtained in real-time.

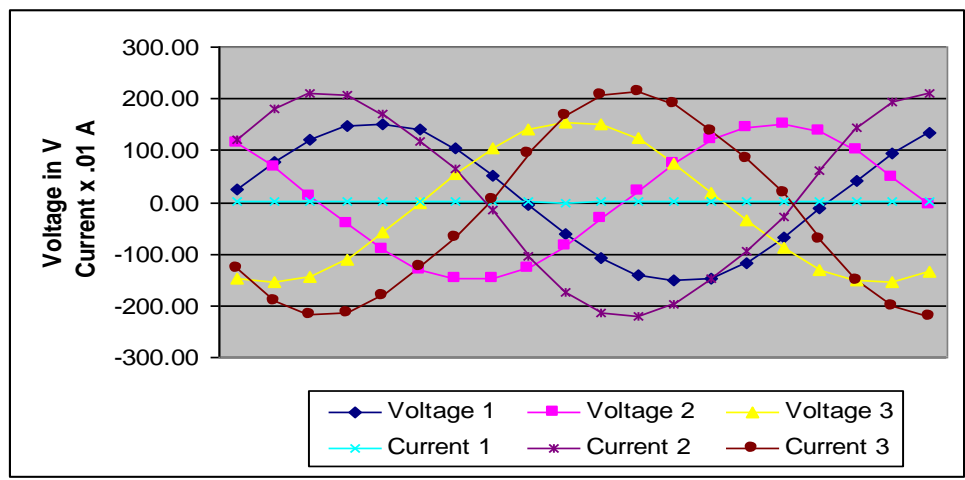

Fig. 5 3-phase voltages and currents for single phasing fault 
International Journal of Innovative Research in Electrical, Electronics, Instrumentation and Control Engineering

Vol. 8, Issue 9, September 2020

DOI 10.17148/IJIREEICE.2020.8901

The National Instruments data acquisition system was used to obtain the current and voltage signals samples. The signal conditioner reduced the voltages and converted currents into their corresponding voltages. The conversion range was \pm $10 \mathrm{~V}$, resulting in the scaling factor of $41.283 \mathrm{~V} / \mathrm{V}$ for voltages and $2.3741 \mathrm{~A} / \mathrm{V}$ for currents to voltages. As in [10], a LabVIEW program acquired instantaneous currents and voltages which were used to calculate RMS values. These RMS currents and voltages are used to train and test the LSTM-based fault detection method. Table 1 lists the number of datasets used to train for each fault type [10]. These consist of 788 cases for various faults. MATLAB software is used to train the LSTM with these datasets [25]. The trained LSTM is tested with the dataset used for training and a set of 21 data that are not used for training. The details of these training and testing results are presented in the next section.

Table 1 Training data cases

\begin{tabular}{|l|c|}
\hline \multicolumn{1}{|c|}{ Condition } & Instances \\
\hline No fault - F1 & 154 \\
\hline Single phasing - F2 & 85 \\
\hline Unbalanced voltage - F3 & 450 \\
\hline Under voltage - F4 & 49 \\
\hline Over voltage - F5 & 10 \\
\hline Locked rotor - F6 & 10 \\
\hline Overload - F7 & 30 \\
\hline Total instances & 788 \\
\hline
\end{tabular}

\section{TRAINING AND TESTING RESULTS FOR IDENTIFYING INDUCTION MOTOR FAULTS}

The proposed model of the LSTM method for induction motor faults identification is shown in Fig. 6. The RMS values of voltage and current inputs are given to LSTM layer as sequence input after proper data preparation.

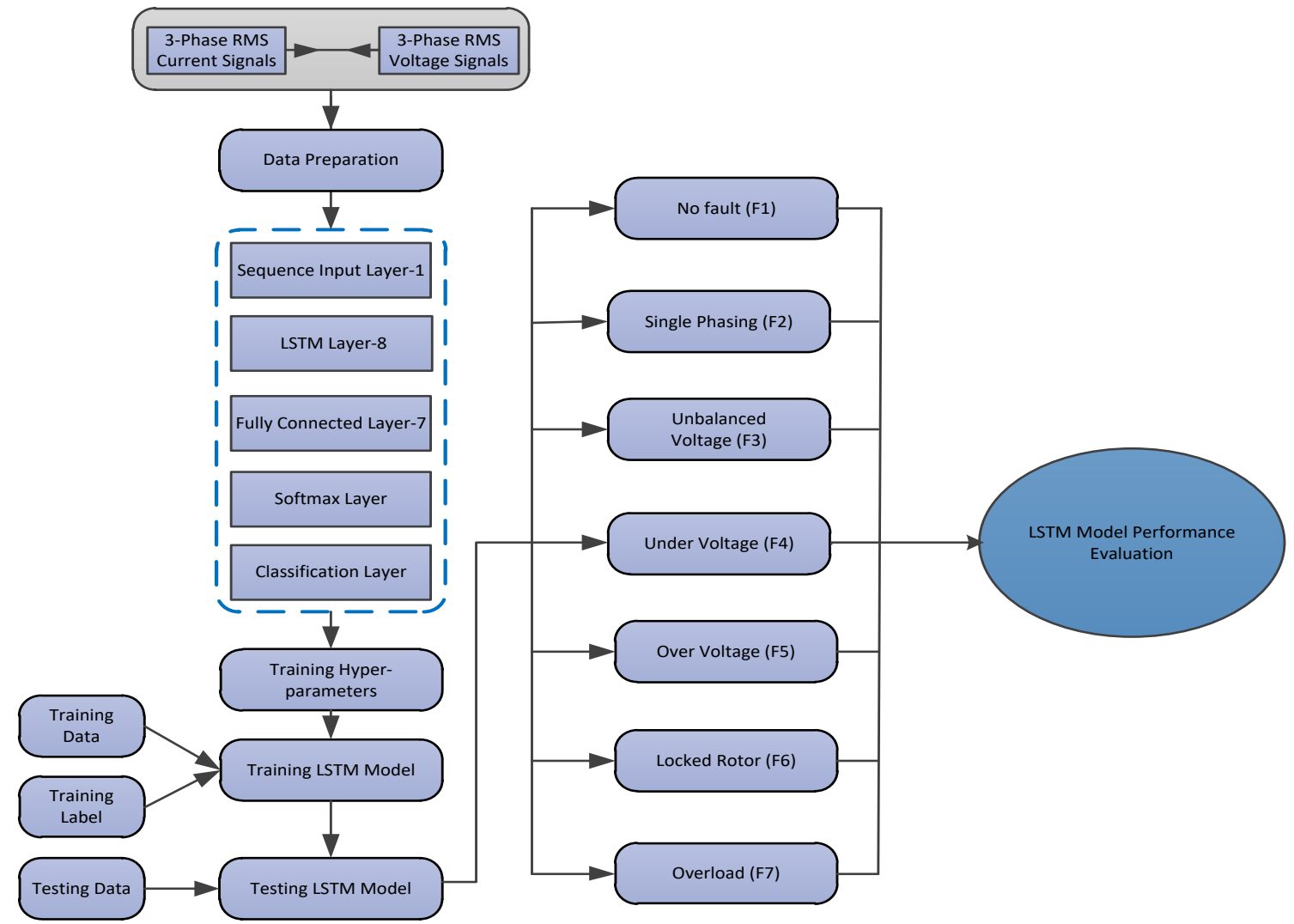

Fig. 6 Proposed model for induction motor faults detection

For training, the 788 faults input data listed in Table 1 are converted into appropriate format for MATLAB. Various other layers needed for LSTM method, indicated in Fig. 3, follow the LSTM layer as shows in Fig. 6. The number of hidden units in LTSM layer is varied from 7 to 20 and set at 8 to achieve the required training performance. The fully connected 
International Journal of Innovative Research in Electrical, Electronics, Instrumentation and Control Engineering

Vol. 8, Issue 9, September 2020

DOI 10.17148/IJIREEICE.2020.8901

layer is set at 7 to correspond to the faults and no faults conditions of induction motor. The parameters used in LSTM method are listed in Table 2 for achieving better performance in the training and testing. As indicated, the Adam optimizer is chosen for its performance. The number of epochs is set as 50 because the accuracy is stable. The learning rate is chosen as 0.1 to achieve the required training performance.

Table 2 Parameters used in LSTM method

\begin{tabular}{|l|c|}
\hline \multicolumn{1}{|c|}{ Parameters } & Value \\
\hline Input Size & 1 \\
\hline Number of Hidden Units & 8 \\
\hline Number of Classes & 7 \\
\hline Epoch & 50 \\
\hline Mini Batch Size & 27 \\
\hline Optimizer & Adam \\
\hline Learning Rate & 0.1 \\
\hline Gradient Threshold & 1 \\
\hline
\end{tabular}

The accuracy and loss for trained model are shown in Fig. 7. The figure displays training progress, elapsed time, number of epochs, number of iterations, iterations per epoch, maximum iterations, type of learning rate schedule and learning rate. From this figure, it is clearly seen that accuracy of the trained model is reached at about $100 \%$ and loss function is also closed to zero after 40 epochs.

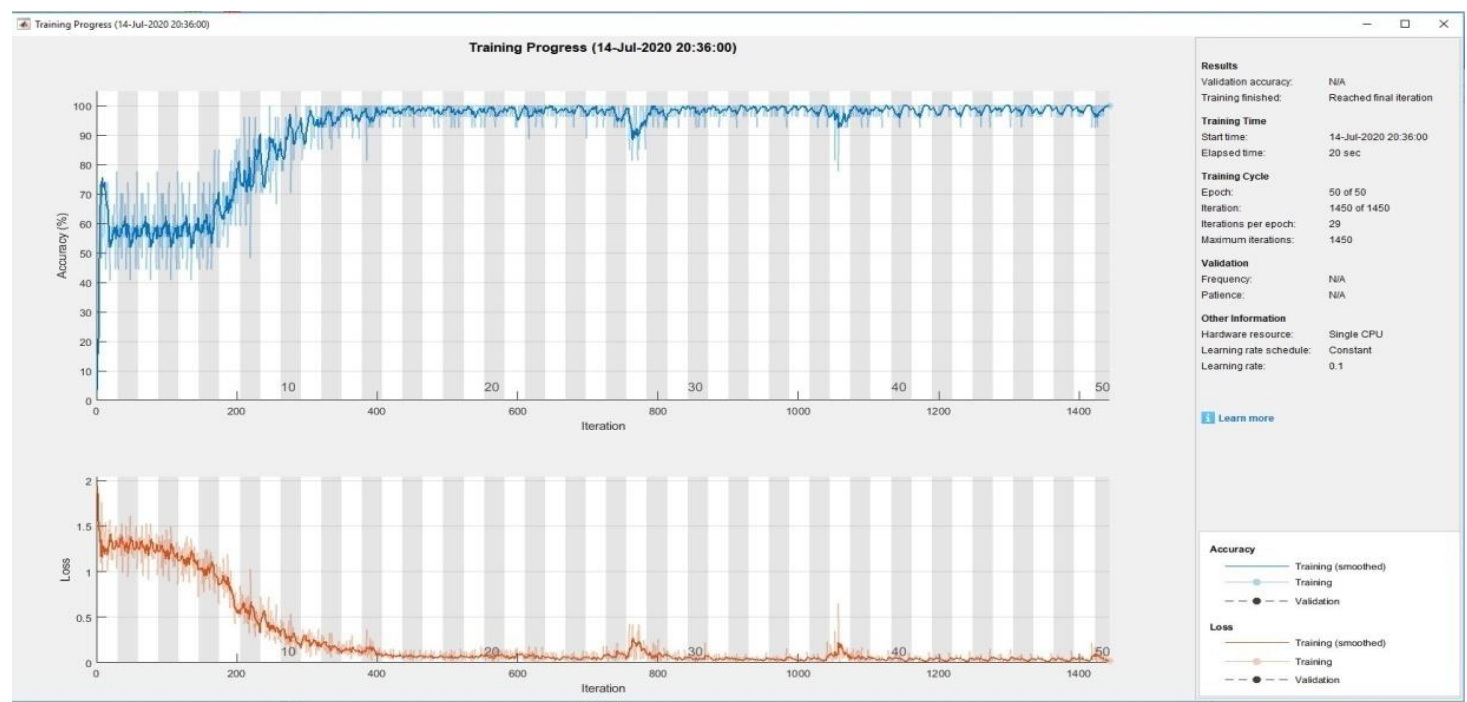

Fig. 7 The accuracy and loss in LSTM fault detection method for 50 epochs of training progress

Table 3 shows the confusion matrix for 788 training data set. In this confusion matrix, it can be seen that trained model predicts all faults and no fault condition correctly achieving 100\% accuracy. The accuracy is calculated using the following equation:

$\mathrm{ACC}=\frac{\mathrm{TP}+\mathrm{TN}}{\mathrm{TP}+\mathrm{FN}+\mathrm{FP}+\mathrm{TN}}$

where ACC is classification accuracy, TP is true positive, TN is true negative, $\mathrm{FP}$ is false positive and FN is false negative value.

Table 3 Confusion matrix for trained data validation

\begin{tabular}{|c|c|c|c|c|c|c|c|c|c|}
\hline \multirow{2}{*}{$\mathrm{n}=788$} & \multicolumn{9}{|c|}{ True Class } \\
\hline & Faults & F1 & $\mathrm{F} 2$ & F3 & $\mathrm{F} 4$ & F5 & F6 & F7 & Accuracy \\
\hline \multirow{7}{*}{ 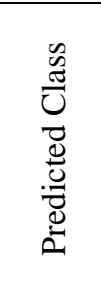 } & F1 & 154 & 0 & 0 & 0 & 0 & 0 & 0 & $100.00 \%$ \\
\hline & $\mathrm{F} 2$ & 0 & 85 & 0 & 0 & 0 & 0 & 0 & $100.00 \%$ \\
\hline & F3 & 0 & 0 & 450 & 0 & 0 & 0 & 0 & $100.00 \%$ \\
\hline & F4 & 0 & 0 & 0 & 49 & 0 & 0 & 0 & $100.00 \%$ \\
\hline & F5 & 0 & 0 & 0 & 0 & 10 & 0 & 0 & $100.00 \%$ \\
\hline & F6 & 0 & 0 & 0 & 0 & 0 & 10 & 0 & $100.00 \%$ \\
\hline & F7 & 0 & 0 & 0 & 0 & 0 & 0 & 30 & $100.00 \%$ \\
\hline
\end{tabular}


International Journal of Innovative Research in

Electrical, Electronics, Instrumentation and Control Engineering

Vol. 8, Issue 9, September 2020

DOI 10.17148/IJIREEICE.2020.8901

The input current and voltage waveforms for one of the cases used to test the LSTM method are shown in Figs. 1, 5, 8 and 9. Fig. 1 depicts the current and voltage waveforms from an unbalanced voltage fault and Fig. 5 shows the waveforms from a single phasing fault. Fig. 8 shows the waveforms for a no fault case and Fig. 9 shows an overload fault case. It can be observed from Fig. 8 and 9 that the 3-phase currents amplitudes are more in overload case than the no fault case. Similar waveforms were obtained for under voltage, over voltage and locked rotor cases [10]. RMS values of currents and voltages for all cases are calculated and reported in Table 4. The third row for each of the cases in the table corresponds to some of these seven currents and voltages waveform figures. The table also shows another two sets of data that are used as a part of the test data for the LSTM method. The confusion matrix for this test data is shown in Table 5. As it can be observed from the table, the LSTM method has correctly identified all the seven types of faults and no fault conditions for these three sets of test data giving an accuracy of 100\%, calculated using Equation (20).

It can be observed that the SVM method in [3] requires selection of kernel functions and scaling of data in training and testing which is not required in LSTM method for fault diagnosis. The ANN method in [10] requires the output to be considered either 1 or 0 within 5\% of their values in identifying the faults whereas the proposed LSTM method does not require rounding for results, making the LSTM method accuracy better than ANN based method [10].

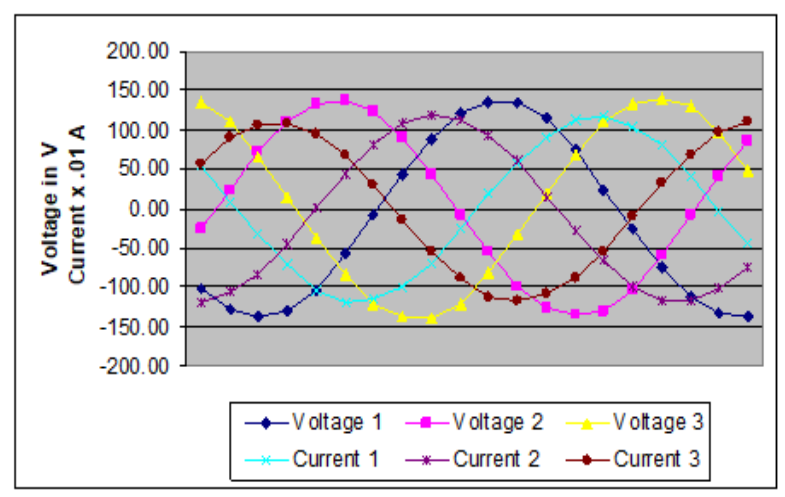

Fig. 8 Voltages and currents for no fault condition

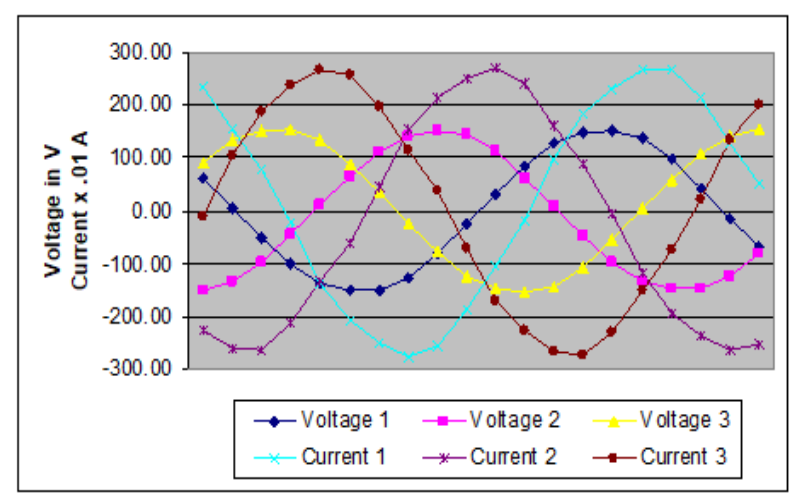

Fig. 9 Voltages and currents for overload fault

Table 4 Data used for testing the LSTM method

\begin{tabular}{|c|c|c|c|c|c|c|}
\hline \multirow{2}{*}{ Faults } & \multicolumn{7}{|c|}{ Inputs } \\
\cline { 2 - 7 } & $\mathrm{V} 1$ & $\mathrm{~V} 2$ & $\mathrm{~V} 3$ & $\mathrm{I} 1$ & $\mathrm{I} 2$ & $\mathrm{I} 3$ \\
\hline \multirow{3}{*}{$\begin{array}{c}\text { No } \\
\text { Fault (F1) }\end{array}$} & 2.650 & 2.640 & 2.696 & 0.428 & 0.428 & 0.431 \\
\cline { 2 - 7 } & 2.694 & 2.652 & 2.690 & 0.517 & 0.521 & 0.502 \\
\cline { 2 - 7 } & 2.622 & 2.614 & 2.666 & 0.410 & 0.418 & 0.417 \\
\hline \multirow{3}{*}{$\begin{array}{c}\text { Single } \\
\text { Phasing (F2) }\end{array}$} & 2.688 & 2.609 & 2.721 & 0.625 & 0.629 & 0.013 \\
\cline { 2 - 7 } & 2.694 & 2.642 & 2.703 & 0.644 & 0.648 & 0.004 \\
\cline { 2 - 7 } & 2.616 & 2.600 & 2.642 & 0.628 & 0.632 & 0.004 \\
\hline \multirow{3}{*}{$\begin{array}{c}\text { Unbalanced } \\
\text { Voltage (F3) }\end{array}$} & 2.624 & 1.906 & 2.022 & 0.538 & 0.249 & 0.332 \\
\cline { 2 - 7 } & 1.682 & 2.475 & 2.702 & 0.186 & 0.634 & 0.628 \\
\cline { 2 - 7 } & 1.624 & 2.019 & 2.585 & 0.207 & 0.449 & 0.576 \\
\hline \multirow{3}{*}{ Under } & 1.088 & 1.087 & 1.084 & 0.231 & 0.239 & 0.225 \\
\cline { 2 - 7 } Voltage (F4) & 1.966 & 1.969 & 1.977 & 0.305 & 0.318 & 0.300 \\
\cline { 2 - 7 } & 1.976 & 1.967 & 1.995 & 0.306 & 0.312 & 0.307 \\
\hline \multirow{3}{*}{ Over } & 2.888 & 2.878 & 2.863 & 0.482 & 0.499 & 0.496 \\
\cline { 2 - 7 } Voltage (F5) & 2.856 & 2.839 & 2.871 & 0.478 & 0.477 & 0.467 \\
\cline { 2 - 7 } & 2.882 & 2.872 & 2.868 & 0.488 & 0.484 & 0.459 \\
\hline \multirow{2}{*}{$\begin{array}{l}\text { Locked } \\
\text { Rotor (F6) }\end{array}$} & 2.657 & 2.613 & 2.687 & 1.671 & 1.651 & 1.669 \\
\cline { 2 - 7 } & 2.649 & 2.614 & 2.647 & 3.090 & 3.096 & 3.043 \\
\cline { 2 - 7 } & 2.573 & 2.565 & 2.609 & 3.052 & 3.073 & 2.996 \\
\hline \multirow{3}{*}{\begin{tabular}{c} 
Overload (F7) \\
\cline { 2 - 7 }
\end{tabular}} & 2.638 & 2.602 & 2.675 & 0.807 & 0.788 & 0.800 \\
\cline { 2 - 7 } & 2.604 & 2.600 & 2.643 & 0.882 & 0.893 & 0.884 \\
\hline
\end{tabular}


IJIREEICE

International Journal of Innovative Research in

Electrical, Electronics, Instrumentation and Control Engineering

Vol. 8, Issue 9, September 2020

DOI 10.17148/IJIREEICE.2020.8901

Table 5 Confusion matrix for testing data

\begin{tabular}{|c|c|c|c|c|c|c|c|c|c|}
\hline \multirow{2}{*}{$\mathrm{n}=21$} & \multicolumn{9}{|c|}{ True Class } \\
\hline & Faults & F1 & F2 & F3 & F4 & F5 & F6 & F7 & Accuracy \\
\hline \multirow{7}{*}{ 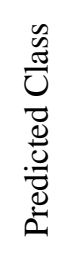 } & F1 & 3 & 0 & 0 & 0 & 0 & 0 & 0 & $100 \%$ \\
\hline & $\mathrm{F} 2$ & 0 & 3 & 0 & 0 & 0 & 0 & 0 & $100 \%$ \\
\hline & F3 & 0 & 0 & 3 & 0 & 0 & 0 & 0 & $100 \%$ \\
\hline & F4 & 0 & 0 & 0 & 3 & 0 & 0 & 0 & $100 \%$ \\
\hline & F5 & 0 & 0 & 0 & 0 & 3 & 0 & 0 & $100 \%$ \\
\hline & F6 & 0 & 0 & 0 & 0 & 0 & 3 & 0 & $100 \%$ \\
\hline & F7 & 0 & 0 & 0 & 0 & 0 & 0 & 3 & $100 \%$ \\
\hline
\end{tabular}

VI. CONCLUSION

The LSTM method is applied for identifying external faults in a 3-phase induction motor in this paper. The paper gives details of various parameters used in the training of the LSTM method. It also gives testing results using fault data obtained from a 1/3 hp 3-phase induction motor. From the results, it can be noted that the testing accuracy of LSTM method is $100 \%$ in identifying all faults and no-fault condition. The performance of the LSTM method is observed to be better than ANN method [10] in accuracy and SVM method [3] in model formation. The paper uses RMS values of currents and voltages as inputs. Other signals that may be considered in future studies are directly using the instantaneous values of currents and voltages.

\section{REFERENCES}

[1]. W. A. Elmore, Protective Relaying: Theory and Applications vol. 1: CRC press, 2003.

[2]. A. G. Phadke and J. S. Thorp, Computer Relaying for Power Systems: John Wiley \& Sons, 2009.

[3]. S. Kolla and R. Hammo, "Support vector machines for fault identification in three-phase induction motors," International Journal of Engineering Research and Innovation, vol. 8, p. 16, 2016.

[4]. M. A. Wani, et al., Advances in Deep Learning: Springer Nature Singapore Pbt Ltd., 2020.

[5]. L. Zhang, et al., "A review on deep learning applications in prognostics and health management," IEEE Access, vol. 7, pp. 162415-162438, 2019.

[6]. A. Siddique, et al., "A review of stator fault monitoring techniques of induction motors," IEEE Transactions on Energy Conversion, vol. 20, pp. 106-114, 2005

[7]. M. Ojaghi, et al., "Diagnosis methods for stator winding faults in three-phase squirrel-cage induction motors," International Transactions on Electrical Energy Eystems, vol. 24, pp. 891-912, 2014.

[8]. M. E. H. Benbouzid and G. B. Kliman, "What stator current processing-based technique to use for induction motor rotor faults diagnosis?," IEEE Transactions on Energy Conversion, vol. 18, pp. 238-244, 2003.

[9]. M.-Y. Chow, et al., "A neural network approach to real-time condition monitoring of induction motors," IEEE Transactions on Industrial Electronics, vol. 38, pp. 448-453, 1991.

[10]. S. R. Kolla and S. D. Altman, "Artificial neural network based fault identification scheme implementation for a three-phase induction motor," ISA Transactions, vol. 46, pp. 261-266, 2007.

[11]. S. Pöyhönen, et al., "Coupling pairwise support vector machines for fault classification," Control Engineering Practice, vol. 13, pp. 759-769, 2005.

[12]. S. B. Salem, et al., "Support vector machine based decision for mechanical fault condition monitoring in induction motor using an advanced Hilbert-Park transform," ISA Transactions, vol. 51, pp. 566-572, 2012.

[13]. O. Uyar and M. Cunkas, "Design of fuzzy logic based motor protection system," in 6th International Advanced Technologies Symposium (IATS'11), 2011, pp. 16-18.

[14]. H. Keskes, et al., "Broken rotor bar diagnosis in induction machines through stationary wavelet packet transform and multiclass wavelet SVM," Electric Power Systems Research, vol. 97, pp. 151-157, 2013.

[15]. R. Fang and H. Ma, "Application of MCSA and SVM to induction machine rotor fault diagnosis," in 2006 th World Congress on Intelligent Control and Automation, 2006, pp. 5543-5547.

[16]. H. Pan, et al., "An improved bearing fault diagnosis method using one-dimensional CNN and LSTM," J. Mech. Eng, vol. 64, pp. 443-452, 2018.

[17]. L. Eren, "Bearing fault detection by one-dimensional convolutional neural networks," Mathematical Problems in Engineering, vol. $2017,2017$.

[18]. J.-H. Lee, et al., "Fault diagnosis of induction motor using convolutional neural network," Applied Sciences, vol. 9, p. 2950, 2019.

[19]. R. Yang, et al., "Rotating machinery fault diagnosis using long-short-term memory recurrent neural network," IFAC-PapersOnLine, vol. 51, pp. 228-232, 2018

[20]. J. Lei, et al., "Fault diagnosis of wind turbine based on Long Short-term memory networks," Renewable Energy, vol. 133, pp. 422-432, 2019.

[21]. S. Hochreiter and J. Schmidhuber, "Long Short-Term Memory," Neural Computation, vol. 9, pp. 1735-1780, $1997 / 11 / 01$ 1997.

[22]. F. A. Gers, et al., "Learning to forget: continual prediction with LSTM," presented at the 9th International Conference on Artificial Neural Networks: ICANN '99, Edinburgh, UK, 1999.

[23]. S. Zhang, et al., "Data-Based Line Trip Fault Prediction in Power Systems Using LSTM Networks and SVM," IEEE Access, vol. 6, pp. 76757686, 2018

[24]. D. P. Kingma and J. L. Ba, "Adam: A Method for Stochastic Optimization," in Published as a conference paper at the 3rd International Conference for Learning Representations, San Diego, 2015.

[25]. Mathworks. (2020). Deep Learning Toolbox. Available: https://www.mathworks.com/products/deep-learning.html. 